\title{
Application Adapter
}

National Cancer Institute

\section{Source}

National Cancer Institute. Application Adapter. NCI Thesaurus. Code C73536.

A software layer that converts data from an application into a form that will allow integ ration with other applications. 